\title{
Das Koriotom - ein neues chirurgisches Gerät zur Präparation von Vollhauttransplantaten
}

\author{
R. Kasten \\ V. Voigtländer
}

\author{
The Coriotome - A New Surgical Device for Preparation of Full-Thickness \\ Skin Grafts
}

\section{Zusammenfassung}

Vollhauttransplantate müssen nach ihrer Entnahme sorgfältig vom subkutanen Fett befreit werden, um die nutritive Versorgung im Empfängerbett nicht zu gefährden. In der Praxis wird gewöhnlich eine zusätzliche Koriumschicht abgetragen, um ein weniger anspruchsvolles Transplantat zu erhalten und Stufenbildungen gegenüber der Umgebung zu vermeiden. Die übliche Präparationsmethode, bei der die Vollhaut mit der Unterseite nach oben z. B. über einen Zeigefinger gelegt oder auf einer Unterlage fixiert und mit einer Schere oder einem Skalpell ausgedünnt wird, ist zeitaufwändig und führt häufig zu Unebenheiten. Bei Unachtsamkeit kann außerdem das Transplantat perforiert werden. Wir stellen ein neues chirurgisches Gerät vor, mit dem das subkutane Fett und variierbare Anteile des Koriums zeitsparend und tiefendefiniert abgetragen werden können. Es resultiert ein Transplantat mit gleichmäßiger Schichtdicke und planer Unterfläche.

\section{Abstract}

Full-thickness skin grafts have to be defatted to improve graft take and to avoid differences in thickness between the graft and the recipient site. The conventional method of thinning the graft with surgical scissors or a scalpel often leaves an irregular surface and is rather time consuming. Especially with a very thin preparation there is a risk of perforating the graft. We present a new surgical device which allows trimming a graft evenly in a considerably shorter period of time.

\section{Einleitung}

Vor der Transplantation von Vollhaut, die definitionsgemäß aus Epidermis mit vollständigem Korium besteht, muss das anhängende subkutane Fett entfernt werden, welches ein Hindernis für eine ausreichende nutritive Versorgung des Transplantats im Wundbett und für neue vaskuläre Verbindungen darstellen würde. Sehr häufig wird zusätzlich ein Teil des Koriums abgetragen, um ein anspruchsloseres Transplantat zu erhalten oder um die Transplantatdicke an die Defekttiefe anzupassen und das Risiko für eine postoperative Polsterbildung der verpflanzten Haut zu verringern [3]. Im engeren Sinne sind diese Transplantate keine Vollhauttransplantate, sondern dicke Spalthauttransplantate. Sie werden allerdings viel häufiger als „echte“ Vollhauttrans- plantate eingesetzt und im klinischen Alltag auch als „Vollhauttransplantate“ bezeichnet.

Üblicherweise wird für die Abtragung von subkutanem Fett und Teilen des Koriums ein Skalpell oder eine Schere verwendet, wobei das Transplantat mit der Unterseite nach oben z. B. über einen Zeigefinger (Abb.1) oder eine gerollte Kompresse gelegt wird [4]. Bei diesem Verfahren ist es jedoch schwierig, das Gewebe gleichmäßig abzutragen. Meist bleibt ein welliges Korium zurück mit dickeren Transplantaträndern, da hier sehr vorsichtig präpariert werden muss, um das Transplantat nicht auszufransen oder zu perforieren. Außerdem kann eine starke Manipulation des Gewebes zu einer Traumatisierung und damit zu einer schlechteren Einheilung führen [8].

Institutsangaben

Hautklinik am Klinikum der Stadt Ludwigshafen (Direktor: Prof. Dr. med. V. Voigtländer)

Widmung

Herrn Prof. Dr. E. G. Jung zum 70. Geburtstag gewidmet.

Korrespondenzadresse

Dr. med. R. Kasten · Hautklinik am Klinikum Ludwigshafen · Bremserstraße 79 · 67063 Ludwigshafen 


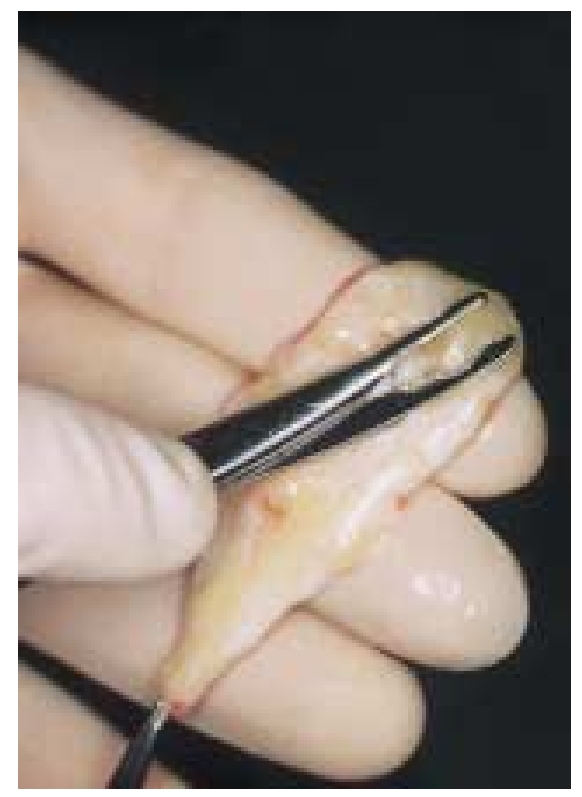

Abb. 1 Herkömmliche Präparation eines Vollhauttransplantats mit der Schere.

\section{Methode}

Mit dem neu entwickelten und „Koriotom“ benannten Gerät (Abb. 2) können das subkutane Fettgewebe und Teile des Koriums tangential flächig und zeitsparend abgetragen werden. Die Abtragungstiefe wird mit dem Winkel, mit dem der Hobel über das Transplantat geführt wird, reguliert. Je spitzer der Winkel, desto tiefer dringt die Klinge ein und desto mehr Gewebe wird abgetragen. Dieser Schneidevorgang wird möglich durch die ellipsoide Form der seitlichen Abstandhalter, an denen der Klingenschlitten schwenkbar aufgehängt ist.

Für die Präparation wird das entnommene Transplantat mit der Unterseite nach oben auf eine glatte, feste Oberfläche, z. B. eine Kunststoff- oder Glasplatte, gelegt und an einem Ende je nach Größe mit einer oder besser 2 Gefäßklemmen fixiert (Abb. 3,6). Die Klemmen sollten das Transplantat rechtwinklig zur Abtragungsrichtung unter Spannung halten. Das Koriotom wird nun in einem Winkel von z. B. $80^{\circ}$ auf das Transplantat in Nähe der Befestigung aufgesetzt. Dann wird die Abtragungstiefe durch Absenkung des Griffes gewählt und das Koriotom in konstantem Winkel vom Fixationspunkt weg über das Transplantat geführt (Abb. 4, 7). Bei Bedarf kann dieser Vorgang mit kleineren Abtragungswinkeln zur zusätzlichen Ausdünnung wiederholt werden. Es resultiert ein Transplantat mit einer glatten, planen Unterfläche in der gewünschten Dicke (Abb. 5, 8).

\section{Diskussion}

Bereits in der Vergangenheit wurde nach technischen Möglichkeiten gesucht, die Ausdünnung von Vollhauttransplantaten zu beschleunigen und das Präparationsresultat zu verbessern. Ein Verfahren, das ohne nachträgliche Manipulation am Transplantat auskommt, ist seine freihändige Gewinnung in vivo mit einem Skalpell [7,8]. Hierbei wird das Transplantat tangential mit einem Skalpell in einer korialen Ebene exzidiert, so dass sich eine Abpräparation des subkutanen Fetts und der tieferen Teile des Koriums erübrigt. Der Hebedefekt muss allerdings nachgeschnitten werden oder sekundär heilen. Außerdem besteht die Gefahr,

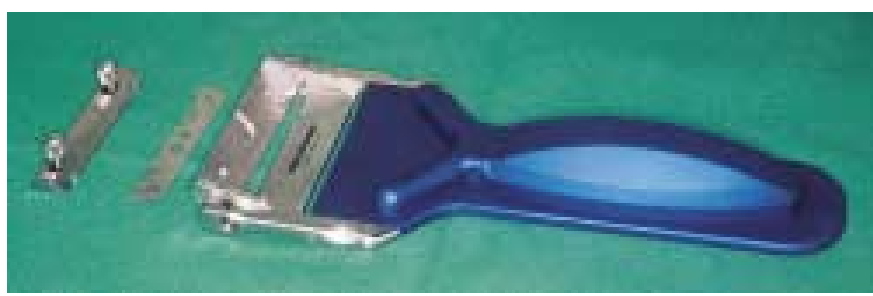

Abb. 2 Koriotom - an einem Griffstück, das sich zu zwei ellipsoiden Abstandhaltern verjüngt, ist ein schwenkbarer Klingenschlitten aufgehängt. Klinge und Klingenhalter sind demontiert.

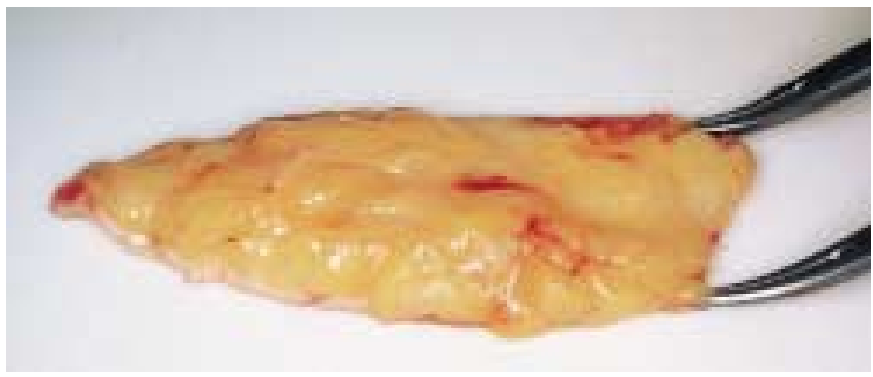

Abb. 3 Exzidat von inguinal $(4,5 \times 9,5 \mathrm{~cm})$ fixiert mit 2 Gefäßklemmen, subkutanes Fettgewebe obenliegend.

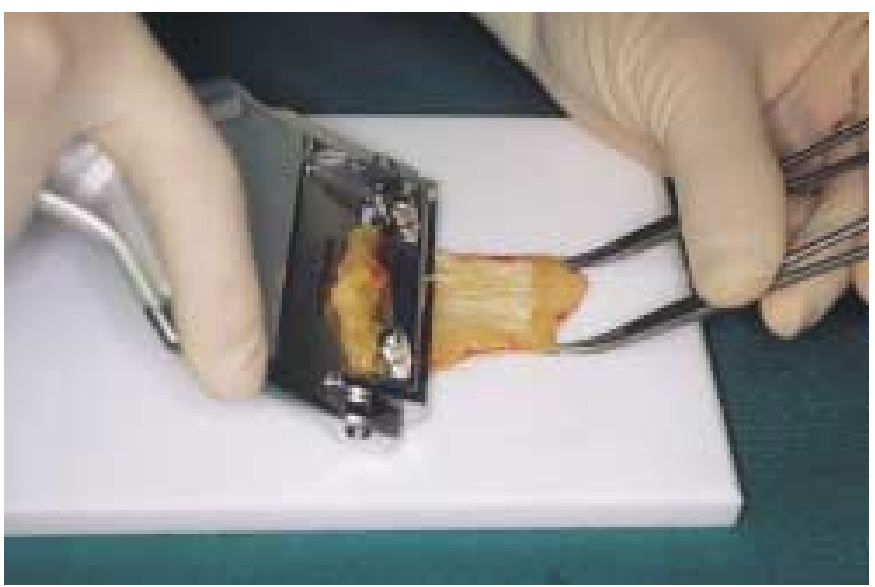

Abb. 4 Tangentiale Abtragung der Subkutis.

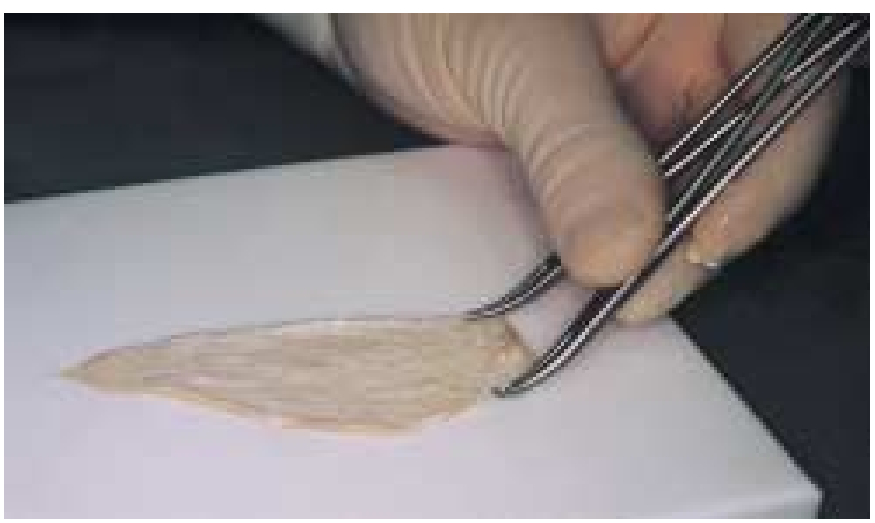

Abb. 5 Exzidat von inguinal nach Abtragung der Subkutis und Teilen des Koriums. 


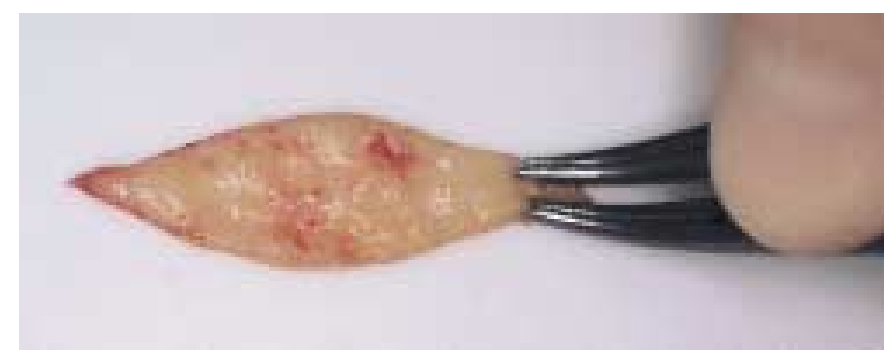

Abb. 6 Exzidat von präaurikulär $(1,5 \times 3,5 \mathrm{~cm})$, Subkutis obenliegend.

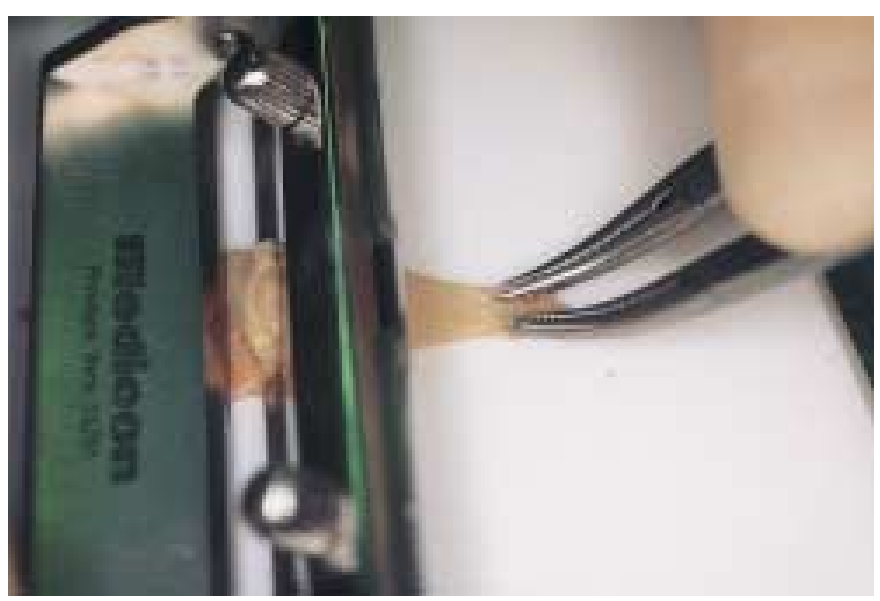

Abb. 7 Tangentiale Abtragung von Subkutis und tiefer Anteile des Koriums.

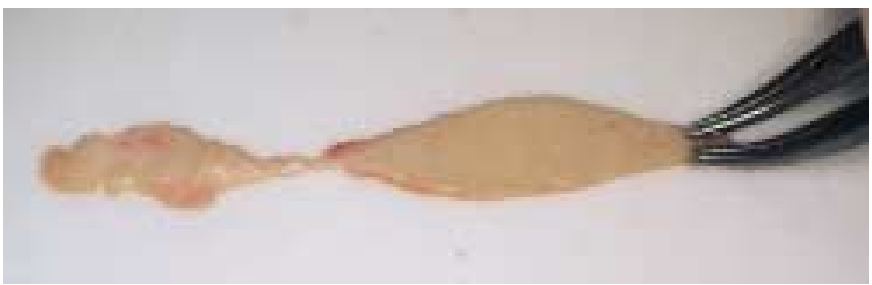

Abb. 8 Glattes, planes Korium nach tangentialer Präparation mit dem Koriotom.

dass das Transplantat mit einer unregelmäßigen Unterfläche entnommen oder perforiert wird.

Konventionelle Dermatome sind zur Ausdünnung von Hauttransplantaten schlecht geeignet, da bei ihnen die Klinge ohne Abstandhalter zur Unterlage die tiefste Ebene darstellt, so dass das Transplantat bei einer Entfernung von subkutanem Fett und Teilen des Koriums leicht durchschnitten werden kann. Eine Ausnahme bilden die „Trommeldermatome“ nach Reese oder Padgett, die zur Gewinnung von Spalthaut v.a. von konvexen Oberflächen, wie der Oberschenkelzirkumferenz, aber auch von abdominal und thorakal geeignet sind [5]. Bei diesen Dermatomen schneidet die Klinge die Haut, die an der Außenseite des trommelförmigen Griffes mit Klebstoff haftet. Mit ihnen ist es auch möglich, die Subkutis und tiefe Dermis eines entnommenen Transplantats zu entfernen, nachdem es mit der epidermalen Seite nach unten auf die Dermatomtrommel geklebt wurde [5]. Aufgrund ihrer umständlichen Handhabung, der selten gleichmäßigen Schichtdicke der Spalthaut und der beträchtlichen Ver- letzungsgefahr für Operateur und OP-Personal werden die Trommeldermatome heute nur noch selten eingesetzt.

Siegert und Jäger $(1994,1998)$ beschrieben ein Verfahren, das die Verwendung eines herkömmlichen Dermatoms ermöglichen soll. Das Transplantat wird hierbei durch Unterdruck auf einem Gitter fixiert und dann von höhenverstellbaren Platten oder unterschiedlich hohen Abstandsrahmen umgeben, mit denen die Eindringtiefe der Dermatomklinge reguliert werden kann $[2,6]$.

Gibson und Ross (1965) stellten ein chirurgisches Gerät vor, mit dem es möglich ist, große Hautareale, die z.B. bei einem Unfall abgeledert worden sind, vor der Replantation schneller zu entfetten. Dieses modifizierte Dermatom verfügt über seitliche $\mathrm{Ab}$ standhalter, die über eine Stellschraube höhenreguliert werden können [1]. Das Gerät ist allerdings für die Präparation von kleineren Transplantaten nicht geeignet. Da die Abtragung mit einer sägenden Bewegung erfolgt, besteht weiter die Gefahr, dass das Transplantat verrutscht und Zacken in die Transplantatränder geschnitten werden. Die Autoren empfahlen daher, das Transplantat mit einem Gewebekleber auf einer glatten Oberfläche zu fixieren und durch den Assistenten in der Längsachse unter Zug zu halten.

Bei dem von uns vorgestellten Gerät erfolgt die tangentiale Abtragung nicht mit sägenden Bewegungen, sondern in einem einzigen Bewegungsablauf von der Klemme weg hin zum Operateur. Auch der Verzicht auf eine Vorrichtung zur Fixierung der Abtragungstiefe führt nicht zu relevanten Niveauunterschieden, so dass es bei der einfachen Konstruktion des Koriotoms bleiben konnte.

Im Vergleich zur konventionellen Präparation mit Schere oder Skalpell ist mit dem Koriotom eine wesentlich schnellere, gleichmäßigere und tiefendefinierte Abtragung möglich. Die Gefahr, die entnommene Haut zu perforieren, ist auf ein Minimum reduziert. Weiterhin besteht durch die kürzere Traumatisierung mit weniger Handschuhkontakt eine geringeres Kontaminationsrisiko für das Transplantat und eine geringere Verletzungsgefahr für den Operateur.

\section{Literatur}

${ }^{1}$ Gibson T, Ross DS. Dermatome for preparing large skin-grafts from detached skin and fat. Lancet 1965; 30: 252-253

2 Jäger H, Siegert R. Gerät zur subepidermalen Ausdünnung der Haut. Gebrauchsmuster G 9403937.2. Bekanntmachung im Patentblatt des Deutschen Patentamtes vom 22.9.94

${ }^{3}$ Johnson TM, Ratner D, Nelson BR. Soft tissue reconstruction with skin grafting. J Am Acad Dermatol 1992; 27: 151 - 165

${ }^{4}$ Rosenberg L, Mercer D. The preparation of small dermal grafts. Ann Plast Surg 1985; 15: 270-271

${ }^{5}$ Rudolph R, Fisher JC, Ninnemann JL. Skin Grafting. First edition. Boston: Little, Brown and Company, 1979

${ }^{6}$ Siegert R. Hautausdünnungsgerät. Gebrauchsmuster DE 29722914 U1. Bekanntmachung im Patentblatt des Deutschen Patentamtes vom 26.3.1998.

${ }^{7}$ Snow SN, Stiff M, Lambert D, Tsoi C, Mohs FE. Freehand technique to harvest partial-thickness skin to repair superficial facial defects. Dermatol Surg 1995; 21: $153-157$

${ }^{8}$ Snow SN, Zweibel S. Freehand skin grafts using the shave technique. Arch Dermatol 1991; 127: 633-635 